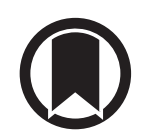

CrossMark

\title{
Gadofosveset-enhanced lung magnetic resonance imaging to detect ongoing vascular leak in pulmonary fibrosis
}

\author{
To the Editor:
}

Vascular leak is a cardinal response to tissue injury $[1,2]$. When dysregulated, vascular leak has been shown to contribute to the development of pulmonary fibrosis in the bleomycin mouse model [3]. Specifically targeting vascular endothelial growth factor, initially described as vascular permeability factor [4] and a key mediator regulating capillary permeability, attenuates the development of pulmonary fibrosis in vivo [5]. Gadofosveset (Ablavar; Lantheus Medical Imaging Inc., North Billerica, MA, USA) is a US Food and Drug Administration-approved, gadolinium-based, albumin-binding contrast agent. Gadofosveset has been used to detect vascular permeability in mouse models [6] and to perform vascular imaging clinically. We hypothesised that gadofosveset-enhanced lung magnetic resonance imaging (MRI) could detect albumin extravasation in subjects with pulmonary fibrosis and demonstrate the location of ongoing tissue injury.

This study was approved though the Partners Institutional Review Board (Partners Healthcare, Somerville, MA, USA) and written consent was obtained. Six subjects with pulmonary fibrosis and four healthy subjects were included. Five subjects had idiopathic pulmonary fibrosis (IPF) and one subject had scleroderma-associated interstitial lung disease with a usual interstitial pneumonia (UIP) pattern. Subjects were excluded for congestive heart failure, pneumonia within 6 weeks of study entry, cigarette smoking within 6 months of study entry, or contraindications to undergoing MRI or receiving gadolinium.

MRI was performed using a commercial 3-T MRI scanner (SKYRA; Siemens Healthcare, Boston, MA, USA) with an 18-channel body array and 12-channel spine array. Images were obtained during free breathing, and acquired using pointwise encoding time reduction with radial acquisition with a flip angle of $25^{\circ}$, echo time $0.050 \mathrm{~ms}$, repetition time $2.24 \mathrm{~ms}, 40 \mathrm{~K}$ radials, three-dimensional field of view of $400 \mathrm{~mm}$ and pixel resolution of $1.56 \times 1.56 \times 1.56 \mathrm{~mm}$. Baseline imaging was performed followed by intravenous injection of gadofosveset at single dose of $0.03 \mathrm{mmol} \cdot \mathrm{kg}^{-1}$ and imaging was repeated for up to 32.5 min post-injection.

Images were analysed using the freeware Dicom reader OsiriX (Pixmeo SARL, Bernex, Switzerland). For each subject, regions of interest (ROIs) were drawn in the ascending and descending aorta at five different standardised locations, two of which were in coronal planes and three of which were in axial planes. ROIs were drawn by hand to outline subpleural regions of each lung, excluding the most medial portions. The lung ROIs were specifically chosen to avoid central blood vessels so as not to confound changes in lung parenchyma signal with potential differences in vasculature between IPF and healthy subjects. The mean signal intensity (SI) of each ROI was measured pre-gadofosveset injection and 5-7.5 min, 10-12.5 min and 15-17.5 min after injection of gadofosveset. We calculated our primary outcome measure, the albumin extravasation index (AEI), as a surrogate measure of pulmonary vascular permeability. The AEI is defined as the change in SI in the parenchyma after gadofosveset injection divided by the change in SI in the aorta after gadofosveset injection.

$$
\mathrm{AEI}=\frac{(\text { SI lung post-gadofosveset })-(\text { SI lung pre-gadofosveset })}{(\text { SI aorta post-gadofosveset })-(\text { SI aorta pre-gadofosveset })}
$$

@ERSpublications

Vascular leak is increased diffusely in the lungs in pulmonary fibrosis and is not limited to radiographic areas of disease involvement http://ow.ly/Zz6Z30jb85l

Cite this article as: Montesi SB, Rao R, Liang LL, et al. Gadofosveset-enhanced lung magnetic resonance imaging to detect ongoing vascular leak in pulmonary fibrosis. Eur Respir J 2018; 51: 1800171 [https://doi. org/10.1183/13993003.00171-2018]. 

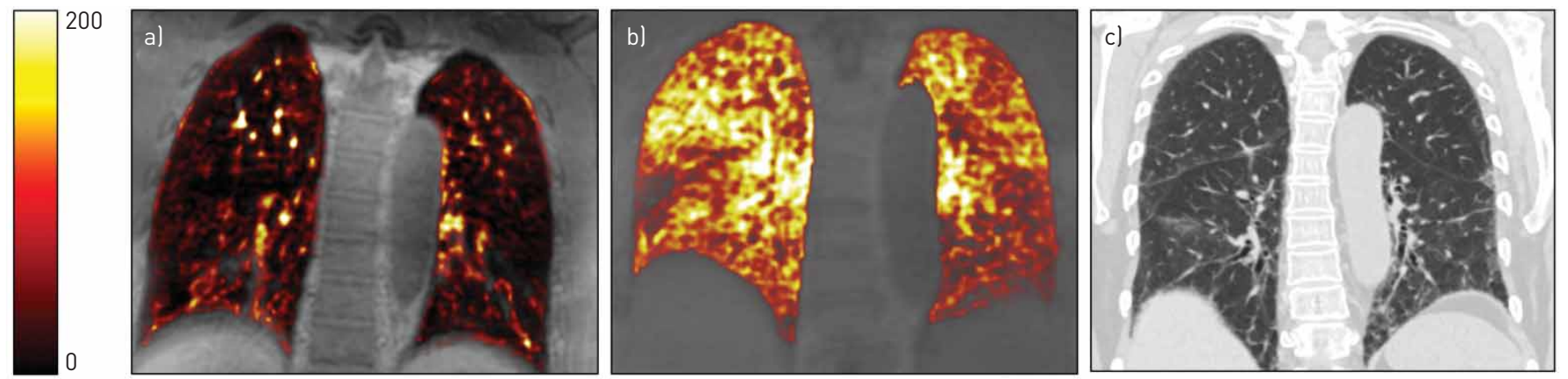

FIGURE 1 Coronal subtraction images with colour overlay. Subtraction of baseline pre-gadofosveset image from 15 min post-gadofosveset image (colour) superimposed on baseline image. a) Healthy control. b) Subject with idiopathic pulmonary fibrosis (IPF). c) Computed tomography image of the same subject with IPF. In a), signal enhancement is isolated to areas of known blood vessels with the majority of the lung unenhanced. In b), regions of signal enhancement extend well beyond major vessels and into the lung parenchyma, consistent with increased albumin extravasation. When comparing b) to cl, regions of increased signal intensity occur in areas of radiographically normal lung in addition to areas with known fibrosis.

We averaged the AEIs from the right and left lung at each location and then across the three time points to use for subsequent calculations. We calculated total, coronal and axial AEIs to assess the degree of vascular permeability from measured lung regions, and AEIs for each anatomical section to assess the anatomical distribution of alveolar-capillary permeability. Statistical analyses were performed using Prism 6.0 (GraphPad Software, La Jolla, CA, USA) using Mann-Whitney U and one-way ANOVA as appropriate, with p-values $<0.05$ considered statistically significant. Data are reported as median (range). To visualise the location of albumin extravasation, subtraction images with colour overlay were constructed (figure 1).

Subjects with pulmonary fibrosis had a total AEI of 0.37 (0.32-0.46) versus 0.17 (0.12-0.28) for controls $(\mathrm{p}=0.01)$. The AEI was increased in subjects with IPF across coronal $(0.34(0.30-0.42)$ versus $0.16(0.13-$ $0.26), \mathrm{p}=0.01)$ and axial $(0.43(0.32-0.56)$ versus $0.20(0.06-0.30), \mathrm{p}=0.01)$ measurements. The AEI was also increased across both anterior $(0.39(0.26-0.45)$ versus $0.15(0.07-0.22), \mathrm{p}=0.01)$ and posterior $(0.32$ $(0.26-0.39)$ versus $0.18(0.16-0.30), \mathrm{p}=0.02)$ coronal locations as well as upper $(0.45(0.40-0.51)$ versus 0.21 (0.10-0.32), $\mathrm{p}=0.01)$, middle $(0.40(0.31-0.57)$ versus $0.21(0.05-0.30), \mathrm{p}=0.01)$ and lower $(0.44(0.33-0.56)$ versus $0.20(0.08-0.30), \mathrm{p}=0.01)$ axial locations in IPF subjects. The AEI was increased similarly across axial locations for UIP subjects. Significance for all AEIs persisted even when excluding the sclerodermaUIP values.

Subtraction images demonstrate a marked increase in SI in IPF patients compared to healthy controls (e.g. figure 1). For the healthy control (figure 1a), the majority of the lung is nonenhanced and high signal enhancement areas are limited to areas of the vascular tree consistent with the expected distribution of a blood pool magnetic resonance contrast agent. For the subject with IPF (figure 1b), however, regions of enhancement extend well beyond major vessels, were located diffusely throughout the lung fields, and did not display a basilar nor a subpleural preference. When compared to computed tomography chest imaging (figure 1c), increased signal intensity occurred in radiographically normal lung as well as areas with fibrotic changes.

We present the novel application of gadofosveset-enhanced MRI to detect pulmonary vascular leak by directly imaging albumin extravasation for the first time. We found that vascular leak was increased in the lungs of individuals with pulmonary fibrosis compared to healthy controls. These findings build on previous observations indicating disruption of the alveolar-capillary membrane in IPF [7-9]. By specifically imaging albumin extravasation from the vascular compartment, our results provide potentially important information about the spatial distribution of ongoing lung injury in pulmonary fibrosis.

Gadofosveset is a small molecule gadolinium-based contrast agent that binds reversibly to serum albumin. After administration, about $80-90 \%$ of the contrast agent is bound to albumin with a dissociation constant of $90 \mu \mathrm{M}$ [10]. We hypothesised that in vascular leak, the extravascular, extracellular albumin concentration will increase due to extravasation of albumin, and the unbound gadofosveset would extravasate rapidly from the blood into the lung interstitial space and bind to albumin present there. In the absence of gadofosveset extravasation, the AEI would equal the blood volume fraction in the region of lung tissue analysed. Higher AEI values would indicate vascular leak. While we did not validate our albumin extravasation index with albumin extravasation histologically, gadofosveset has been used to assess vascular permeability and response to treatment in the setting of mouse models of accelerated atherosclerosis with correlation of vessel wall MRI signal changes with Evans blue dye [6]. 
Albumin extravasation was increased in subjects with pulmonary fibrosis similarly across all regions of the lung. Our results suggest that lung injury occurring in pulmonary fibrosis is more diffuse than traditionally thought, and this finding questions why basilar fibrosis predominates in conditions like IPF. Other factors, such as tractional stress or collapse induration, as have been hypothesised [11-13], may create regional differences in wound healing responses that can promote fibrosis rather than normal repair. Insight into the mechanobiological drivers of fibrosis and signalling pathways upregulated by stretch, including transforming growth factor- $\beta[14]$, may elucidate this further. Albumin extravasation was not limited to fibrotic regions and occurred in areas of radiographically normal lung. Whether some component of these findings could be explained by very early fibrotic changes or neovascularisation is not known. Lung regions with increased vascular permeability may represent areas at risk of developing radiographically apparent fibrosis. Additional research is needed to assess associations between the amount of vascular leak and disease progression. Our results represent an important step towards the use of molecular imaging to assess known processes directly involved in the development of fibrosis.

Sydney B. Montesi ${ }^{1}$, Rohan Rao ${ }^{1}$, Lloyd L. Liang ${ }^{1}$, Hannah E. Goulart ${ }^{1}$, Amita Sharma ${ }^{2}$, Subba R. Digumarthy ${ }^{2}$, Barry S. Shea ${ }^{3}$, Ravi T. Seethamraju ${ }^{4,5}$, Peter Caravan ${ }^{2,5,6}$ and Andrew M. Tager ${ }^{\dagger 1}$

${ }^{1}$ Division of Pulmonary and Critical Care Medicine, Massachusetts General Hospital and Harvard Medical School, Boston, MA, USA. ${ }^{2}$ Dept of Radiology, Massachusetts General Hospital and Harvard Medical School, Boston, MA, USA. ${ }^{3}$ Division of Pulmonary, Critical Care and Sleep Medicine, Albert Medical School of Brown University, Providence, RI, USA. ${ }^{4}$ Siemens Healthcare, Boston, MA, USA. ${ }^{5}$ Athinoula A. Martinos Center for Biomedical Imaging, Massachusetts General Hospital, Charlestown, MA, USA. ${ }^{6}$ Institute for Innovation in Imaging, Massachusetts General Hospital, Charlestown, MA, USA.

Correspondence: Sydney B. Montesi, Division of Pulmonary and Critical Care Medicine, Massachusetts General Hospital, 55 Fruit Street, BUL-148, Boston, MA 02114, USA. E-mail: sbmontesi@partners.org.

Received: Jan 252018 | Accepted after revision: March 062018

Acknowledgements: We would like to gratefully acknowledge the important contributions from Mary O'Hara and Larry White of the Athinoula A. Martinos Center for Biomedical Imaging (Massachusetts General Hospital, Charlestown, MA, USA).

Conflict of interest: S.B. Montesi reports receiving grants from the Parker B. Francis Foundation, the National Institutes of Health, Harvard Catalyst and the Chest Foundation, during the conduct of the study. B.S. Shea reports receiving grants from the National Institutes of Health/NHLBI during the conduct of the study; and consulting fees from Boehringer Ingelheim and Roche-Genentech, and grants from Biogen, outside the submitted work. R.T. Seethamraju is an employee of Siemens Healthineers, the manufacturer of MRI machines on which this study was performed. In addition, he has two patents, "Free-breathing Radial-MR Angiography with No Contrast (FRANC)" and "Non-contrast Breath Held MR Perfusion of the Lungs from Time Series Analysis", pending to Siemens and Brigham and Women's Hospital. P. Caravan reports receiving grants from the National Institutes of Health during the conduct of the study; and personal fees and nonfinancial support from Collagen Medical LLC, nonfinancial support from Factor 1A LLC and Reveal Pharmaceuticals, grants from Pfizer and Pliant, and personal fees from Bayer and Guerbet, outside the submitted work.

Support statement: This work was conducted with support from Harvard Catalyst, The Harvard Clinical and Translational Science Center (National Center for Research Resources and the National Center for Advancing Translational Sciences, National Institutes of Health Award UL1 TR001102), and financial contributions from Harvard University and its affiliated academic healthcare centres. The content is solely the responsibility of the authors and does not necessarily represent the official views of Harvard Catalyst, Harvard University and its affiliated academic health centres, or the National Institutes of Health. This work was also supported in part by grants from the National Institutes of Health (F32HL129789 to S.B. Montesi, K08HL105656 to B.S. Shea, and R01HL131907 to P. Caravan and A.M. Tager) and the Parker B. Francis Foundation (S.B. Montesi). Funding information for this article has been deposited with the Crossref Funder Registry.

\section{References}

Singer AJ, Clark RA. Cutaneous wound healing. N Engl J Med 1999; 341: 738-746.

2 Dvorak HF. Tumors: wounds that do not heal: similarities between tumor stroma generation and wound healing. N Engl J Med 1986; 315:1650-1659.

3 Shea BS, Brooks SF, Fontaine BA, et al. Prolonged exposure to sphingosine 1-phosphate receptor-1 agonists exacerbates vascular leak, fibrosis, and mortality after lung injury. Am J Respir Cell Mol Biol 2010; 43: 662-673.

4 Senger DR, Galli SJ, Dvorak AM, et al. Tumor cells secrete a vascular permeability factor that promotes accumulation of ascites fluid. Science 1983; 219: 983-985.

5 Iyer AKV, Ramesh V, Castro CA, et al. Nitric oxide mediates bleomycin-induced angiogenesis and pulmonary fibrosis via regulation of VEGF. J Cell Biochem 2015; 116: 2484-2493.

6 Phinikaridou A, Andia ME, Protti A, et al. Noninvasive magnetic resonance imaging evaluation of endothelial permeability in murine atherosclerosis using an albumin-binding contrast agent. Circulation 2012; 126: 707-719.

7 Wells AU, Hansell DM, Harrison NK, et al. Clearance of inhaled ${ }^{99 \mathrm{~m}} \mathrm{Tc}-\mathrm{DTPA}$ predicts the clinical course of fibrosing alveolitis. Eur Respir J 1993; 6: 797-802.

8 Mogulkoc N, Brutsche MH, Bishop PW, et al. Pulmonary ${ }^{99 \mathrm{~m}} \mathrm{Tc}-\mathrm{DTPA}$ aerosol clearance and survival in usual interstitial pneumonia (UIP). Thorax 2001; 56: 916-923. 
9 McKeown S, Richter AG, O'Kane C, et al. MMP expression and abnormal lung permeability are important determinants of outcome in IPF. Eur Respir J 2009; 33: 77-84.

10 Eldredge HB, Spiller M, Chasse JM, et al. Species dependence on plasma protein binding and relaxivity of the gadolinium-based MRI contrast agent MS-325. Invest Radiol 2006; 41: 229-243.

11 Leslie KO. Idiopathic pulmonary fibrosis may be a disease of recurrent, tractional injury to the periphery of the aging lung: a unifying hypothesis regarding etiology and pathogenesis. Arch Pathol Lab Med 2012; 136: 591-600.

12 Lutz D, Gazghar A, Lopez-Rodriquez E, et al. Alveolar derecruitment and collapse induration as crucial mechanisms in lung injury and fibrosis. Am J Respir Cell Mol Biol 2015; 52: 232-243.

13 Todd NW, Atamas SP, Luzina IG, et al. Permanent alveolar collapse is the predominant mechanism in idiopathic pulmonary fibrosis. Expert Rev Respir Med 2015; 9:411-418.

14 Froese AR, Shimbori C, Bellaye P-S, et al. Stretch-induced activation of transforming growth factor- $\beta$ 1in pulmonary fibrosis. Am J Respir Crit Care Med 2016; 194: 84-96. 